\title{
EXPERIENCE USING THE IBM SUPPLY CHAIN SIMULATOR
}

\author{
Sugato Bagchi \\ Stephen J. Buckley \\ Markus Ettl \\ Grace Y. Lin \\ IBM T.J. Watson Research Center \\ P.O. Box 218 \\ Yorktown Heights, NY 10598, U.S.A.
}

\begin{abstract}
The IBM Supply Chain Simulator (SCS) is a software tool that can help a company or a group of companies make strategic business decisions about the design and operation of its supply chain. SCS and its predecessors were originally developed by IBM Research to improve IBM's internal supply chains. The tool has played an important role in the resurgence of IBM over the last six years. In 1997 the IBM Industry Solution Units began using the tool to help its clients improve their supply chains. After about a year of business, successful engagements have been completed in a variety of geographies and business segments.

SCS deploys a mix of simulation and optimization functions to model and analyze supply chain issues such as site location, replenishment policies, manufacturing policies, transportation policies, stocking levels, lead times, and customer service. This paper reviews the capabilities of SCS and presents experience from practical studies.
\end{abstract}

\section{INTRODUCTION}

Art Mesher, former Research Director of the Gartner Group, recently made the following comment: "Right now all the systems activity is being done at the execution tactical operations level. You know - make it or fulfill it. What's missing is the ability to truly analyze supply chains over time as a decision-support tool that allows us to understand cause and effect between fundamental issues like inventory warehousing and transportation cost as they relate to customer service and the customers' ability and willingness to pay. What's still missing from the marketplace is the ability to do what-if analyses and supply-chain simulations." [Cooke 1998]

We could not agree more with Mr. Mesher. In fact, many people believe that SCS is the missing link. Our experience is that strategic analysis of supply chain issues using a tool like SCS usually saves tens or hundreds of millions of dollars. The reason is that SCS quantifies the cost of supply chain design options, allowing the lowest cost design to be identified prior to implementation.

We have found that a typical strategic analysis requires a combination of optimization and simulation. Optimization technology such as linear or mixed integer programming is useful for solving well-defined mathematical problems such as warehouse location and inventory optimization. But these problems are rigidly defined and usually have simplifying assumptions in their formulations. Such problems must be solved independently and thus in a complex supply chain one must decompose all of the issues into a hierarchical sequence of problems. At best, a hierarchical approach is awkward and introduces a second level of simplifying assumptions. At worst, this approach may not be feasible if some of the problems are nonlinear or stochastic. SCS resolves this situation by cleanly integrating optimization algorithms into an end-to-end supply chain simulation. The simulation environment can be used to test the optimizers under more realistic assumptions. Moreover, SCS is general-purpose across supply chain domains.

Supply chain simulation differs from traditional types of discrete event simulation. For example, it differs from traditional manufacturing simulation because it spans far beyond the four walls of a manufacturing site. Its goal is to improve the financial position of an entire enterprise or a group of trading partners. Supply chain simulation is much more tuned to cost than traditional manufacturing simulation, and the cost drivers come from all areas of an enterprise. The existing body of manufacturing simulators cannot be re-deployed to solve strategic supply chain problems without a significant amount of redesign. 


\section{OVERVIEW OF THE IBM SUPPLY CHAIN SIMULATOR}

SCS is an analytical software tool that emerged from internal IBM modeling and reengineering studies [Feigin et al 1996, Buckley 1996, Buckley and Smith 1997]. The tool offers a unique combination of graphical process modeling, discrete event simulation, animation, activitybased costing, and optimization.

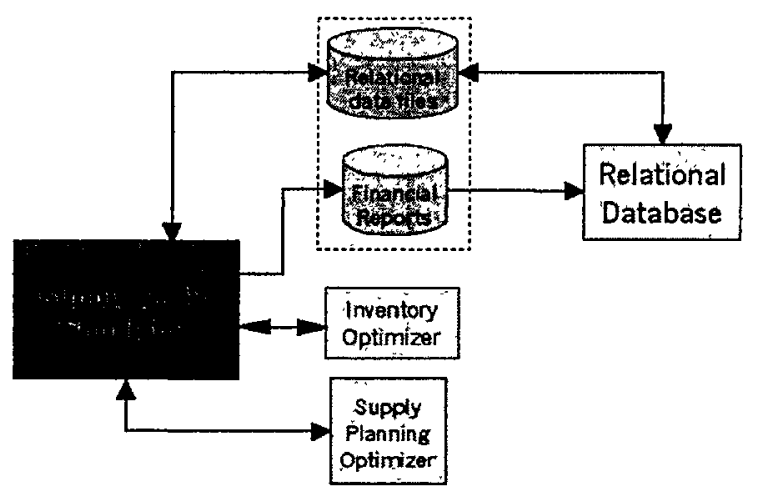

Figure 1: System Architecture of the Supply Chain Simulator

Figure 1 shows the SCS system architecture. SCS is built upon SIMPROCESS [Swegles 1997], a general purpose business process simulator. SIMPROCESS is a product of CACI Products Company. SCS preserves all SIMPROCESS capabilities while offering additional supply chain functionality. Although SCS offers a completely self-contained user interface, flat files in relational format offer an alternative way to specify model inputs. These files can be imported and exported from spreadsheets or relational databases. Additional relational flat files containing detailed financial reports are generated after each simulation. These files can also be examined and queried using spreadsheets or relational databases. The relational database also provides a way to import data into SCS from external systems.

\subsection{Modeling Features}

SCS provides modeling functions for seven different supply chain processes:

Customer: This process represents outside customers that issue orders to the supply chain being modeled. Orders are generated on the basis of customer demand, which may be modeled as a sequence of specific customer orders (obtained from historical records) or as an aggregated demand over a period of time. The customer process may also contain information on the desired service level and priority for the customer. When forecasting and supply planning activities are included in a supply chain model, the customer process may issue forecasts of future demand to aid these activities.

Manufacturing. This process models assembly and keeps raw material and finished goods inventory. It can also be used to model suppliers. During simulation, the manufacturing process makes use of modeled information such as, the types of manufactured products, their manufacturing time, bills of material, manufacturing and replenishment policies for components and finished goods, storage capacity, manufacturing and material handling resources, and the order queuing policy.

Distribution. This process models distribution centers, including finished goods inventory and material handling. It can also be used to model a retail store. The inventory replenishment policy, safety stocks, reorder points, material handling resources, storage types and capacities can be modeled for the distribution center or retail store.

Transportation. This process models transportation time, vehicle loading, and transportation costs. Order batching policies (by weight or volume), material handling resources and transportation resources owned by this node may be specified.

Inventory Planning. This process models periodic setting of inventory target levels. Underlying this process is an optimization program developed at IBM Research called the Inventory Optimizer that computes recommended inventory levels at various locations in a supply chain based on desired customer serviceability [Ettl et al 1996].

Forecasting. This process models product forecasts, including promotional and stochastic demand, for future periods.

Supply Planning. This process models the allocation of production and distribution resources to forecast demand under capacity and supply constraints. Underlying this process is another optimization program developed by IBM Research [Dietrich et al 1995].

Figure 2 shows a simple SCS model. A Customer node sends orders to a Distribution Center node. The distribution center processes customer orders and sends finished goods to a Transportation node which ships the goods to the customer. The distribution center needs to replenish its stock from time to time, so it sends replenishment orders to a Manufacturing node that assembles finished goods from supplied parts. The manufacturer in turn replenishes its parts supply by sending orders to two suppliers, both represented by Manufacturing nodes. In this model, the manufacturer and the two suppliers each have their own Transportation node. The customer also sends forecasts to a Forecasting node. The Forecasting node accumulates forecasts and periodically sends them to a Supply Planning node, which creates build plans for the distribution center, manufacturer, and suppliers. 


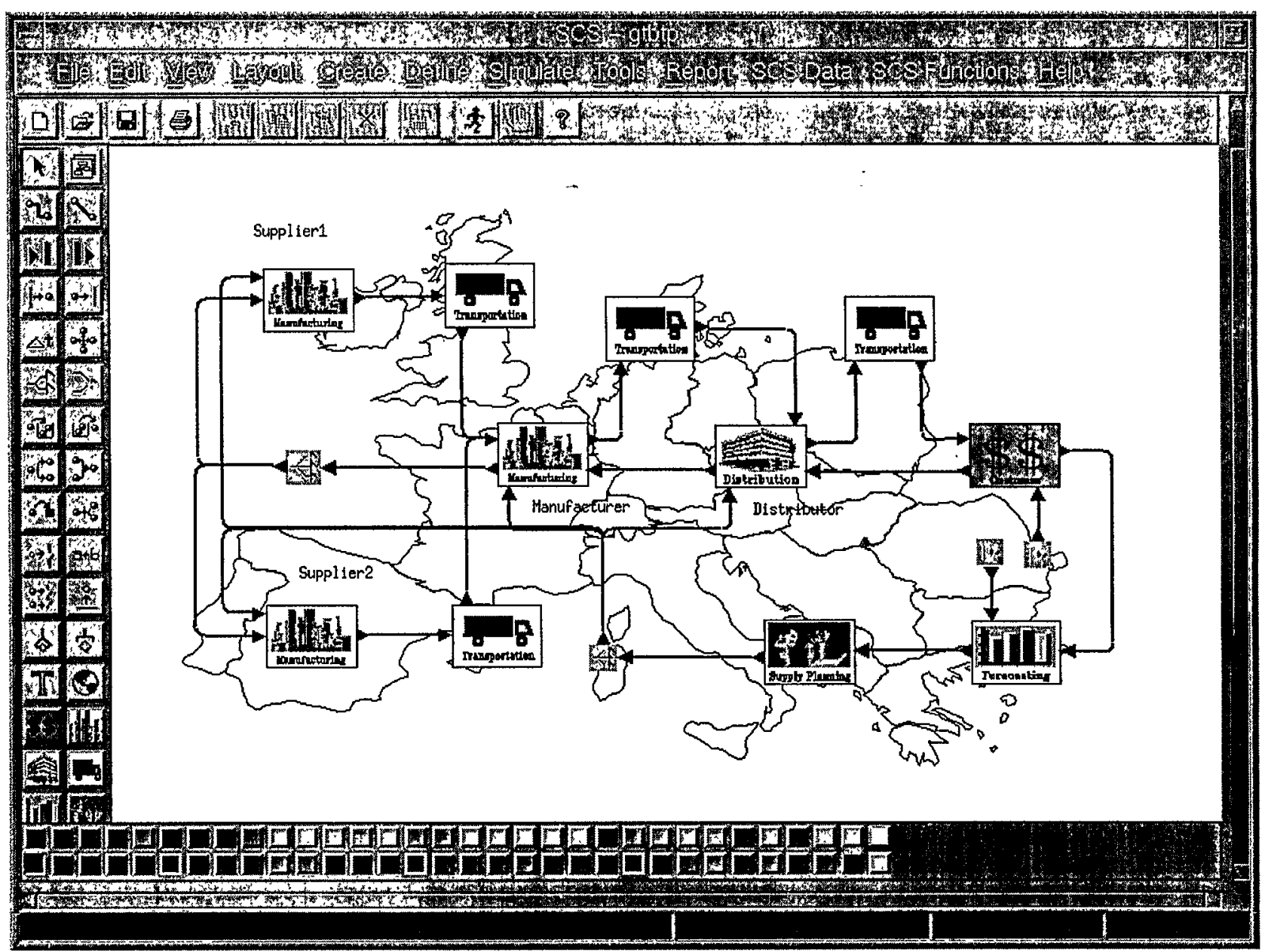

Figure 2: A Simple SCS Model

As with any simulator, SCS allows the user to vary a set of input parameters while monitoring output reports until the best set of output values can be obtained. In a typical supply chain simulation, the following input parameters are varied:

- Number and location of suppliers, manufacturers, and distribution centers

- Stocking level of each product at each site

- Manufacturing and replenishment policies, e.g. Build To Plan (BTP), Build To Order (BTO), Assemble To Order, Continuous Replenishment, Periodic Replenishment

- Transportation policies e.g. Full Truckload (FTL) vs. Less Than Truckload (LTL)

- Supply planning policies e.g. Capacitated vs. Uncapacitated

- Lead times

- Supplier reliability

- Demand variability
To measure each simulation run, numerous reports are available. They include:

- Cycle time, serviceability, fill rate, stockout rate, shipments, and revenue

- Inventory and WIP

- Resource utilization and costs. Resource cost reports include manufacturing (materials, equipment, and labor), transportation, material handling, and inventory holding costs.

- Returns, expired products, unsellable products, incorrect order penalties, and late order penalties

The most important of these reports are the financial reports. The goal is to come up with the minimum cost design that achieves the desired customer serviceability and revenue targets. The financial reports can be thought of as a logistics scorecard for a proposed supply chain design. The next section describes the financial reporting capability of SCS in more detail. 


\subsection{Financial Reports}

SCS makes it possible to evaluate supply chains on the basis of financial tradeoffs associated with various configurations and operational policies. The financial impact may be analyzed from the following items, which are monitored during simulation:

- cost of raw material,

- revenue from goods sold,

- activity-based costs such as material handling and manufacturing,

- inventory holding costs,

- transportation costs,

- cost of expired perishable goods,

- penalties for incorrectly filled or late orders delivered to customers,

- credits for incorrectly filled or late deliveries from suppliers,

- cost of goods returned by customers, and

- credits for goods returned to suppliers.

These reports are generated by four major financial components within SCS. These are: activity based costing, inventory costing, transportation costing, and intercompany financial transactions.

\subsubsection{Activity Based Costing}

The simulation of activities modeled using SCS allows the monitoring of the cost drivers such as replenishment orders, shipments, returns, and cancellations. These drivers require the performance of activities, which in turn make use of resources, as defined in the supply chain model. Activity based costing (ABC) [Turney 1991] is the process of assigning the cost of using resources to the activities that make use of them, and then assigning the cost of an activity to the cost objects for which the activity was performed. The cost objects relevant to supply chains include companies, orders, and products. The cost assignments are based on the time-weighted usage of the resources by activities and the activities by orders, which are monitored during the course of the simulation.

$A B C$ is performed on the basis of the resource costs provided in the model. Resources, such as material handling personnel, assemblers, and manufacturing equipment, have fixed and variable costs. Fixed costs, such as salaries and depreciation, are those that do not depend on amount of time for which the resource is used. Variable costs, such as hourly rates and energy costs are proportional to time or usage frequency. SCS allows for this information to be included for each modeled resource.
At the end of a simulation, the $A B C$ reports detail the average and total costs by cost period, resource, activity, order type, product, company, and strategies used for distribution and replenishment. The cost assignment is computed using both capacity and full absorption costing algorithms.

\subsubsection{Inventory Holding Costs}

Supply chain design decisions are often made to optimize inventory. The cost of holding inventory is therefore an important statistic. This cost is incurred in the form of warehouse depreciation or rental costs as well as interest payments on the value of the inventory (when the inventory is financed through loans).

SCS generates inventory holding cost reports in a manner similar to that for $A B C$. The warehouse cost is distributed over the inventoried products in proportion to the quantity of the products and the duration for which they were held. Often, a warehouse has various kinds of storage facilities (refrigerated, dry, etc.) with differing operating costs. This can be modeled in SCS by specifying the types and capacity of storage facilities for a manufacturing or distribution center, with the appropriate fixed and variable costs for each facility. An annual interest rate (if applicable) may also be specified for the inventory.

The inventory holding cost report consists of the holding cost broken down by cost period, storage facility, manufacturing or distribution center, product, and company that owns the inventory. In addition to the inventory holding cost, the time-weighted average quantity and value of the inventory (broken down along the same dimensions) is reported as well.

\subsubsection{Transportation Costs}

The cost of transporting goods from one location to another may arise from various sources. The use of a vehicle and personnel may incur an activity-based cost. The in-transit inventory may also incur an inventory holding cost. Finally, a shipper may also charge a cost for transporting the goods depending on the route taken. SCS allows the modeling of all these sources of transportation costs. The activity-based and inventory holding costs are described above. The route-based cost may be modeled by defining a route and specifying one or more of the following costs: the full truckload cost and the less than truckload cost per unit weight or volume. Shippers often make the distinction between full and partially filled trucks for cost computation. The full truckload cost is typically less than shipping an equivalent amount over multiple trucks. This information may be used to determine the optimal shipment sizes and the extra costs when the size varies from the optimal. 


\subsubsection{Financial Transactions}

When goods are shipped from one company to another during the simulation of a supply chain in SCS, the corresponding financial transaction is recorded. The type of transaction may be a fulfilled order, return, penalty due to late or incorrectly filled order, unsellable items, rebates, and expired items. The transaction includes the cost period, type of transaction, sending and receiving companies, product, and the replenishment and distribution strategy (if any) associated with the transaction.

The value of a financial transaction is computed on the basis of the modeled financial information. Revenue and purchase cost calculations are based on product price schedules that may vary by season and volume discounts.

Penalties due to late or incorrectly filled orders are calculated from a company's penalty policy. For late orders, a fixed penalty and/or a value representing a fraction of the price may be charged. For incorrectly filled orders, a company may charge a fixed penalty as well as a variable penalty equal to the amount in error.

\section{THE INVENTORY OPTIMIZER}

Maintaining the right level of inventory at the right place and the right time helps cost reduction, customer responsiveness, and supply chain efficiency. Excess inventory incurs extra holding costs, storage costs, storage space, depreciation, price-take-down, and possibly salvage costs. On the other hand, inventory shortage causes poor customer serviceability, excess non-square set of component inventory, and worse yet, loss of business to competitors. Inventory level is a key decision associated with supply chain performance. Due to the size and complexity of the problems, it is often impossible to use the simulation what-if analysis approach to find an optimal solution for inventory optimization and allocation. A computer or food industry application can involve hundreds to thousands of part numbers with multi-level bills-of-materials and widely distributed lead time and cost, tens to hundreds manufacturing and distribution sites, cross docking, supplier, and customer locations linked by different transportation modes. The complexity involves the tight coupling of the inter-relationship among inventory level and serviceability of different part numbers within the network, the aggregate effect of the variability associated with each step of the delay time and other supply chain uncertainties such as supplier reliability, feature ratio variability, and the highly volatile and difficult to forecast customer demands.

The Inventory Optimizer was developed to address the above issues based on an analytical approach. It models the network inventory optimization problem with serviceability constraints as a constrained nonlinear programming problem. An approximation scheme was developed to estimate the actual lead time of each product by taking into account the upstream parts availability, the associated demand over the lead time, etc. A characterization of the operation at each buffer location was derived based on a one to one continuouis replenishment base-stock inventory policy, using an inventory-queue model. Heuristic search algorithms were derived and implemented to optimize the overall inventory cost while meeting customer service level requirements. The Inventory Optimizer capability includes inventory optimization, calculating "optimal" inventory levels and costs to meet the customer service level requirements in an uncertain environment; serviceability optimization, calculating the "optimal" service levels and recommends inventory allocation when the inventory budget is specified. Input to the Inventory Optimizer consists of product information including product name, build location, lead time, cost, bills-of-materials, usage rate, feature ratios, transit time between two locations, demand forecast and forecast accuracy, and the desired serviceability for each demand stream, or inventory budget. The Inventory Optimizer produces a report describing the results of its optimization including optimal overall inventory cost, or serviceability, annual shipment, turnover, inventory units and cost by part number and by location, work-in-process unit and cost, and the base-stock level.

The Inventory Optimizer can be used as a stand-alone tool for inventory or serviceability optimization. It can also be run before running a simulation to set optimal initial inventory and base-stock levels, or run periodically during the simulation to reset optimal inventory and base-stock levels according to the up-to-date demand forecast. The combined effect of the inventory policy with other supply chain policies such as replenishment policy, transportation mode, network configuration, product structure, and business process can be studied when the Inventory Optimizer is used in conjunction with SCS. The combination of modeling, optimization, and supply chain simulation functionality provides powerful and versatile capabilities to model the dynamic supply chain environment and to perform inventory, serviceability, system parameters, supply chain structure, product structure, and business scenario tradeoff analyses.

\section{EXPERIENCE FROM PRACTICAL STUDIES}

\subsection{The Food Industry}

SCS has been used in several successful consulting studies for the food industry. In each case, we have shown the client how a significant amount of money can be saved by carefully changing the way they do business. In general, our clients do not want their studies to be revealed, to preserve their strategic advantage. In this section we will 


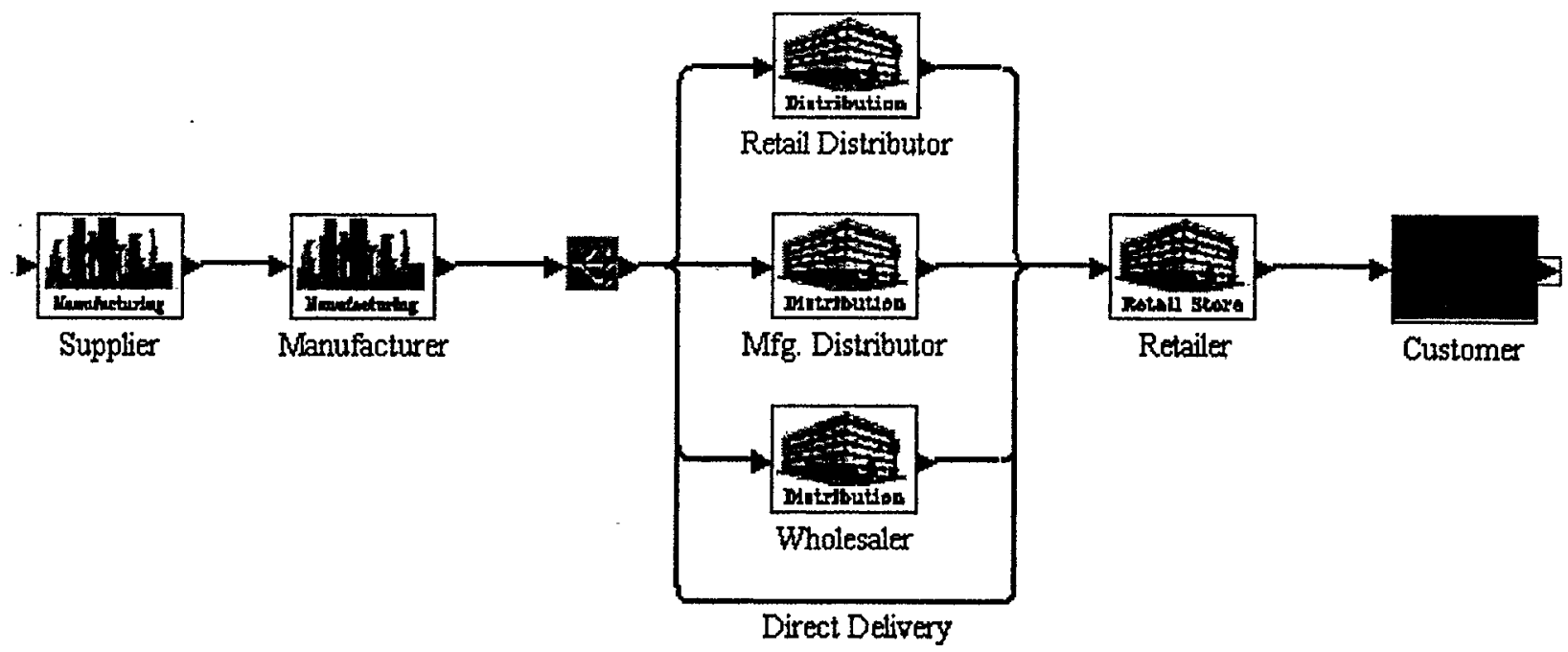

Figure 3: Some Distribution Options in a Food Industry Supply Chain

summarize some of our findings without compromising confidentiality.

We have modeled almost every level of the food industry supply chain starting with the supply of raw materials and ending with the purchase of food products at retail stores. In the middle of the supply chain are food manufacturers, manufacturing distribution centers, retail distribution centers, and wholesalers (see Figure 3). The specific issues we have studied include:

- Continuous Replenishment: We have shown that clients can achieve big savings in inventory by converting from Build-To-Plan to Continuous Replenishment [Buckley and Smith 1997]. Continuous replenishment is commonly used at retail stores but we have also demonstrated the advantage of using it to replenish goods at a distributor or a manufacturer. To model this in SCS, one can specify the replenishment policy for each product at each location. If the replenishment policy is Build-To-Plan, a forecast is specified at the Customer node for that product and its requirements during each time period are propagated down to the Build-To-Plan location by a Supply Planning process (see Figure 2). If the replenishment policy is Continuous Replenishment, a reorder point must be specified for the product. Whenever the onhand and on-order inventory dips below the specified reorder point, a replenishment order is issued to bring the inventory back to the level of the reorder point. The replenishment order that is issued honors a minimum order quantity and a lot size if specified. If desired, the modeler can use the Inventory Optimizer to automatically calculate the reorder points needed to meet the desired customer serviceability.
- Vendor Managed Inventory: We have shown that vendor managed inventory can be a win-win situation for both parties in a supplier/receiver relationship. The receiver can save money from inventory reduction and by outsourcing the management of its replenishment process. The supplier can save money by creating full pallet orders, which greatly reduces material handling costs, and by optimizing transportation costs through creation of full truckload direct manufacturing shipments.

- Cross Docking: The replenishment and material handling policies at a distribution center can be converted from a stockkeeping policy such as Continuous Replenishment to a cross docking or flowthrough policy that keeps no safety stock. We have simulated this transition to see if there are any negative impacts on customer serviceability that offset the positive impacts on inventory levels and storage space costs. With one client we showed that this conversion could be made without loss of customer service. Indirectly, the ability to do this saved them from building new facilities, thereby yielding large savings.

Note that conclusions like these should not be thought of as generally applicable. For each supply chain case study, relevant information must be gathered, modeled, and evaluated through simulation to see if the anticipated conclusions hold. 


\subsection{The Computer Industry}

Another field where SCS has been applied with considerable success is the computer manufacturing and distribution industry. We have redesigned global supply chains by streamlining their supplier and distribution networks, by determining global supply strategies, and by reengineering business processes throughout manufacturing and logistics. We adopt the strategic view that a supply chain is a set of manufacturing sites, suppliers, customers, products, and methods of inventory control, replenishment and distribution. The specific issues we have investigated include:

- Material Requirements Planning (MRP): MRP systems have been used in the computer industry since the 1960's. An MRP system is a centralized system in which inventory is being allocated periodically according to forecasted demand. The timing information needed to schedule order releases is included in the master production schedule. This process can be represented using the supply planning function of SCS. The supply planning function explodes the bills-of-materials to obtain components and raw material requirements that result from the forecasted demand.

- Supplier Integration: To improve order fulfillment, inventory posture and market responsiveness, a division within a large computer manufacturing company considered the re-design of its component sourcing strategy through supplier integration. Supplier integration means that inventory levels and replenishment needs at the assembly site are communicated electronically to all suppliers. The suppliers provide line-side stocking of their components at the assembly site, and the components inventory is being replenished periodically based on actual orders. In constructing a model for supplier integration, we focused on products and manufacturing operations that constitute a representative subset of total goods sold. The manufacturing lead times, transit times, material handling delay times, bills-of-materials, and unit costs were obtained from the company's central database. To characterize the demand for products, we obtained an eight months outlook for each product from the company's forecasting group. The outlook was determined by analyzing historical sales and by collecting predicted future sales from the marketing organization. To carry out the analysis, we reviewed the current business model, which was based on MRP, together with the integrated supplier model in terms of serviceability and inventory performance. We simulated the model under the assumption of weekly replenishment of the line-side component inventory. The reorder points of components and finished goods buffers at the assembly site were determined by the Inventory Optimizer so as to achieve $98 \%$ on-time delivery to end-customers. Assuming that the suppliers who provide line-side stocking own the on-hand component inventory at the assembly site, the simulation results indicated that the division's inventory turnover rises by a factor of greater than two. At the same time, the increase in supplier inventory liability turned out to be so low (less than $10 \%$ ) that it could be compensated for with a small price increase.

- Channel Assembly: The interdependencies between manufacturers and distribution channels were the subject of various simulation studies using SCS. As many computer manufacturers recently started to extend their supply chains to include major distribution channels, there is a need to better understand the impact of different operational policies on total supply chain inventory and serviceability. Among the operational policies we investigated was channel assembly (custom configuration of computers with plug-in features such as memory modules and hard drives), limiting the amount of channel inventory, and the consolidation of distribution channels to regional configuration hubs.

\section{SUMMARY}

We believe that the IBM Supply Chain Simulator represents the leading edge in supply chain simulation. Leading-edge clients and industry consultants have substantiated this belief. We have found SCS to be extremely valuable in practice when costly design and operational decisions are needed in a supply chain.

\section{ACKNOWLEDGMENTS}

Many people contributed to the evolution of the IBM Supply Chain Simulator. Chae An, Dan Connors, Gerry Feigin, Tony Levas, Nitin Nayak, Raja Petrakian, Ramesh Srinivasan, and Jayashankar Swaminathan worked on earlier versions of the software. Richard Shore, Jeffrey Smith, and Bret Naccarato are SCS users who guided the functionality based on client requirements. Tomi Magome, Elizabeth Poole, Margarita Rovira, Bill Tulskie, and Laura Warren made significant contributions to the current software and its documentation. 


\section{REFERENCES}

Buckley, S. 1996. Supply Chain Modeling. Autofact, Detroit, Michigan.

Buckley, S. and Smith, J. 1997. Supply Chain Simulation. Georgia Tech Logistics Short Course, Atlanta, Georgia.

Cooke, J. 1998. A look into the supply chain's future. Logistics Management \& Distribution Report V37 N5 May 1998, 45-48.

Dietrich, B., Connors, D., Ervolina, T., Fasano, J.P., Lin, G., Srinivasan, R., Wittrock, R., Jayaraman, R. 1995. Production and Procurement Planning Under Resource Availability Constraints and Demand Variability. IBM Research Report RC-19948, Yorktown Heights, New York.

Ettl, M., Feigin, G., Lin, G., Yao, D. 1996. A Supply Network Model with Base-Stock Control and Service Requirements. To appear in Operations Research.

Feigin, G., An, C., Connors, D., Crawford, I. 1996. Shape Up, Ship Out. ORMS Today, April.

Swegles, S. 1997. Business process modeling with SIMPROCESS. Winter Simulation Conference, Piscataway, NJ, 606-610.

Turney, P. B. B. 1991. Common Cents: The ABC Performance Breakthrough. Cost Technology, Hillsboro, $\mathrm{OR}$.

\section{AUTHOR BIOGRAPHIES}

SUGATO BAGCHI is a Research Staff Member at the IBM Thomas J. Watson Research Center in Yorktown Heights, NY. His current research interest is in the area of modeling business systems for objectives ranging from strategy formulation to business process design. He has a $\mathrm{Ph} . \mathrm{D}$. degree in Electrical Engineering from Vanderbilt University.

STEPHEN J. BUCKLEY is a Research Staff Member at the IBM Thomas J. Watson Research Center in Yorktown Heights, NY. He is currently the manager of the Supply Chain Analysis department, which developed the Supply Chain Simulator. He received the Ph.D. degree from MIT in Computer Science. In addition to simulation, his interests include algorithms, scheduling, and robotics.

MARKUS ETTL is a Research Staff Member at the IBM Thomas J. Watson Research Center in Yorktown Heights, NY. He received a doctorate degree in Computer Science from the University of Erlangen-Nuernberg. His research interests include supply chain management, network optimization, and quantitative analysis of manufacturing and communication systems.
GRACE Y. LIN is a Research Staff Member at the IBM T.J.Watson Research Center. She is responsible for the development of the Inventory Optimizer and several supply chain modeling projects. She received her Ph.D. degree in Industrial Engineering from Purdue University. Her research interests include extended enterprise supply chain management, inventory management, cooperative control and scheduling, and computer integrated manufacturing. Her publications have appeared in the IJFMS, IIE Transactions, Computer Control of FMS. 